Olga A. Kostrova

Samarskij gosudarstvennyj socialno-pedagogicheskij universitet

IZABELA PROKOP

Uniwersytet im. Adama Mickiewicza w Poznaniu

\title{
Schwerpunkte immer noch schwer? Einige Überlegungen über die deutsche Grammatik für slawische native speakers
}

\author{
Focal points still difficult? \\ Some reflections on German grammar \\ for Slavic native speakers
}

\begin{abstract}
The aim of this article is to present the project of a contrastive Polish-German and Russian-German grammar, which arose as a result of the cooperation between Samara State University of Social Sciences and Education and Adam Mickiewicz University in Poznan. The idea of a unilateral grammar which is based on the categories of the mother tongue of the learner and is aimed at the foreign language to be learned, is designed as a suitable measure within the framework of error prevention. The grammar contains selected grammatical categories that are significant sources of errors for native Slavic speakers.
\end{abstract}

KEYWORDS: Contrastive grammar, foreign language lessons, mistakes, error prevention.

SCHLÜSSELWÖRTER: Kontrastive Grammatik, Fremdsprachenunterricht, Fehler, Fehlerprophylaxe.

\section{ZUR PROBLEMSTELLUNG}

Im vorliegenden Beitrag wollen wir das Konzept einer neuen kontrastiven polnisch-deutschen und russisch-deutschen Grammatik grob skizzieren, weil wir die Ansicht vertreten, dass nur eine von den Kategorien der Muttersprache ausgehende erklärende Grammatik den Lernenden helfen kann, die bis jetzt zahlreichen Fehler zu vermeiden. Alle Lerner auf dem Fortgeschrittenenniveau, ausgebaute Sprachkompetenz, kommunikative Kompetenz, die sich auch im interethnischen Kontakt gut bewährt, selbstsichere und selbstbewusste, fließend 
sprechende Sprachbenutzer, die sich in exolingualer Kommunikation völlig verständlich ausdrücken können, nur sporadisch vorkommende grammatische Fehler - soll es nur ein Traum der überarbeiteten Lehrer und Lektoren sein? Abgesehen von unterschiedlichen Arbeitsformen, in denen die Fremdsprachen gelehrt und gelernt werden, ist die Grammatik ein sehr wichtiger Bestandteil des Fremdsprachenunterrichts an den Universitäten und Hochschulen, insbesondere in der Philologieausbildung. Die Tatsache, dass zahlreiche grammatische Fehler in mündlichen und schriftlichen Äußerungen unserer Studierenden vorkommen, rechtfertigt unsere Idee, eine neue erklärende Grammatik des Deutschen zu verfassen. Die Zusammenarbeit der polnischen und russischen Germanisten hat erlaubt festzustellen, dass unsere Studierenden beim Erwerb des Deutschen als Fremdsprache ähnliche Probleme haben. Es handelt sich aber nicht um unterschiedliche Mängel beim Sprechen, wie zum Beispiel Artikulationsfehler, Führung der Intonationslinie oder das Sprechtempo, sondern vor allem um grammatische Fehler, die sich negativ auf die gesamte Kommunikation in deutscher Sprache auswirken. Bei näherer Betrachtung dieser Fehler stellen wir fest, dass sie einerseits auf die Besonderheiten des Sprachbaus der slawischen Sprachen, andererseits auf die Eigenart der slawischen Kulturen und der slawischen Mentalität zurückzuführen sind. Wie in der Medizin, so auch im Fremdsprachenunterricht sollten wir nach dem Motto „besser vorbeugen als heilen“ handeln und uns in stärkerem Maße mit der Fehlerprophylaxe beschäftigen. Vor allem sollten wir uns die Frage nach den Fehlerursachen stellen.

\section{GLOTTODIDAKTISCHE PRINZIPIEN}

„Man kann, genetisch genommen, die kontrastive Grammatik als ein Nebenprodukt der modernen Theorie des Fremdsprachenunterrichts bezeichnen. In der Praxis des Fremdsprachenunterrichts erkannte man, dass der Lernende die Fremdsprache auf der Basis der Muttersprache sich anzueignen versucht. Das bedeutet, dass der Lernende überall dort, wo wir es mit sprachlichen Elementen der Fremdsprache zu tun haben, welche mit den entsprechenden Elementen der Muttersprache nicht übereinstimmen, diese Elemente als muttersprachliche Elemente zu perzipieren, zu speichern und, was am wichtigsten sein durfte, diese Elemente zu reproduzieren versucht. Diesem Prozess der muttersprachlichen Interferenz unterliegen selbstverständlich vor allem die geschlossenen Strukturen der Sprache“ (Zabrocki 1980: 254). Bis jetzt haben wir es ständig und ununterbrochen mit dem störenden Einfluss der Interferenz ${ }^{1}$ sowohl im Sprach-

${ }^{1}$ Dort, wo Sprachstrukturen in der Ausgangssprache und der zu lernenden Fremdsprache identisch sind, verläuft der Lernprozess ohne Störung. Die strukturellen Unterschiede zwischen 
als auch im Grammatikunterricht zu tun. Unter vielen verschiedenen Faktoren, die das Fehlerentstehen verursachen, nennt Kieweg (2007) auch falsches Nachschlagen im Wörterbuch. In unserem Falle handelt es sich auch um falsches Nachschlagen im Grammatikbuch, wenn ein native speaker des Russischen oder des Polnischen in deutscher Grammatik nach den Ausdrucksmöglichkeiten des slawischen perfektiven Aspekts oder verschiedener Aktionsarten vergebens sucht. Deswegen scheint das Kontrastivitätsprinzip als eine geeignete Maßnahme der Fehlerprophylaxe zu sein, wobei die slawische Muttersprache (Russisch oder Polnisch) als Ausgangssprache, und Deutsch - als Zielsprache dargestellt wird. Erst dann kann man erwarten, dass wenigstens einige Fehlertypen erheblich reduziert werden können. V.B. Kaschkin meint, dass die Berücksichtigung der Muttersprache ökologische linguistische Technologien rechtfertigt (Kashkin 2001: 230), weil die Persönlichkeit und die mentale Welt der Lerner nicht ignoriert werden. Dieselben Annahmen liegen einem Pragmatiklehrbuch zugrunde, es handelt sich um „Pragmatik von Aufforderungshandlungen im Deutschen, Polnischen und Russischen“ von Urszula Kantorczyk, Ewa Komorowska, Irina Lysakova und Tatjana Veselovskaja (2008). Drei Teile des Gesamtvorhabens sind den Aufforderungshandlungen in drei Sprachen gewidmet und bestehen aus der theoretischen Einführung in die Pragmatik der Aufforderungen sowie aus eingehender Beschreibung direktiver Sprachhandlungen, wie Bitte, Rat, Vorschlag, Empfehlung, Anleitung, Forderung und dem darauffolgenden Übungsteil. Wie die Autorinnen im Vorwort deklarieren, „richtet sich das Buch an Personen, für die die interkulturelle Kommunikation zwischen Deutschen, Polen und Russen eine berufliche Notwendigkeit darstellt: an Studierende höherer Semester, die eine oder zwei der drei Sprachen als Fremdsprache erlernen, an Studierende, die ein Auslandssemester in Deutschland, Polen oder Russland absolvieren wollen, an Touristen, die eines der drei Länder kennen lernen und nicht nur im eigenen muttersprachlichen Umfeld kommunizieren wollen sowie an Personen, die berufliche Kontakte mit Firmen in einem dieser Länder anstreben oder vertiefen“ (Kantorczyk 2008: 12). Natürlich stehen hier die situationsadäquaten Sprachhandlungen im Vordergrund, bei ihrer Realisierung aber muss der Lerner zu angemessenen Formeln greifen. In unserem Grammatikbuch dagegen stehen im Interessenzentrum diejenigen grammatischen Kategorien, die in der Ausgangssprache (Muttersprache der Lerner) vorhanden sind und in der Zielsprache (d.h. der ersten oder der zweiten Fremdsprache) fehlen und auf eine andere Art und Weise, z. B. mit Hilfe von lexikalischen Mitteln wiedergegeben werden können.

den Sprachen führen hingegen oft zur Übertragung der Eigenschaften der Grundsprache auf die Zielsprache. So entsteht die Interferenz - das negative Transfer aus der AS auf die ZS - die zu Fehlern führt. 
Sowohl die Grundannahmen der Lernerautonomie als auch die Prinzipien der anthropozentrisch orientierten Glottodidaktik führen uns zur Überzeugung, dass die Frage nach dem Idiolekt der Lerner bei der Planung und Gestaltung des Unterrichts in das Interessenzentrum der Lehrer gerückt werden muss. Den meisten Lernenden fällt es äußerst schwierig, ihre Redebeiträge so zu gestalten, dass sie sich von Äußerungen anderer Mitschüler in der Wahl sprachlicher Mittel unterscheiden. Der Idiolekt des Einzelnen geht im Fremdsprachenunterricht häufig verloren. Die Frage nach dem Idiolekt spielt aber eine sehr wichtige Rolle in der anthropozentrisch orientierten Glottodidaktik, deren Zentrum der Mensch als ein sprach-, wissens- und kulturgenerierendes Wesen darstellt (Grucza 1997: 15).

„Menschliche Sprache ist das praktische Wissen einer jeden Person, auf Grund dessen sie

a) Formen (Strukturen) für Ausdrücke / Äußerungen eines bestimmten Typs bildet und sie substanziert, d.h. sie äußert;

b) bestimmte Ziele mit Hilfe von Ausdrücken / Äußerungen dieses Typs realisiert;

c) ihnen bestimmte Werte zuschreibt, vor allem die Zeichenfunktion;

d) analoge Ausdrücke / Äußerungen erkennen lernt, die von anderen Personen erzeugt werden, d.h. identifiziert und differenziert sie;

e) die in ihnen steckenden Inhalte, vor allem ihre Bedeutung liest und versteht" (Grucza 1993: 31).

Die Ursache dieser „Vereinheitlichung“ von studentischen Äußerungen resultiert unseres Erachtens aus der Tatsache, dass das Repertoire der sprachlichen Mittel häufig allzu begrenzt ist, insbesondere im Bereich von grammatischen Strukturen, die doch eine bestimmte Varianz bieten können. Unser Grammatikbuch soll also den Lernern eine Palette von verschiedenen Strukturen des Deutschen bieten, aus der sie später diejenigen wählen können, die Bestandteil ihres Idiolekts sind. Eine feste Überzeugung, dass man in der Fremdsprache alles ausdrücken kann, was man auch in der Muttersprache vermag, gibt dem Lerner mehr Selbstsicherheit und reduziert seinen Stress. Ein geeignetes Grammatikbuch, das die Schwerpunkte der deutschen Grammatik vom Standpunkt der Muttersprache der Lerner beleuchtet, kann dabei sehr behilflich sein.

Erklärende Grammatik des Deutschen ist auf die Lehrer und Lerner mit slawischen Muttersprachen orientiert und soll das reale Funktionieren der Sprache widerspiegeln. Davon ausgehend, müssen in einer solchen Grammatik zwei Aspekte besonders gewichtet werden. Zum einen ist es die Beschreibung der grammatischen Phänomene in enger Verbindung mit ihren Gebrauchskontexten, in der Schriftsprache sind es Textsorten, in unmittelbarer Kommunikation sind es konkrete Sprachhandlungsmuster, wobei die Parameter kommunikativer Situationen berücksichtigt werden. Zum anderen ist es wichtig, beide Aspekte 
kontrastiv darzustellen, slawische Muttersprachen für Etalonsprachen haltend, an denen Gemeinsamkeiten und Unterschiede gemessen werden, unter Berücksichtigung des Idiolekts und der Idiokultur. Dadurch soll sich das geplante Buch von vorhandenen kontrastiven Grammatiken unterscheiden, zum Beispiel von der deutsch-polnischen kontrastiven Grammatik (Engel et al. 2000), die als „,eine reversible vergleichende Grammatik gedacht <ist>, sie kann in der einen oder in der anderen Richtung benutzt werden" (Engel et al. 2000: 8). Diese unilaterale Spezifik soll als Grundlage für Didaktisierung des Grammatikunterrichts dienen, wobei mögliche Interferenzen der Muttersprachen vermieden bzw. reduziert werden können. Die Lerner sollen sich die Frage stellen: Welche grammatischen Kategorien des Deutschen müssen verwendet werden, um diverse Sachverhalte auszudrücken, die im Russischen bzw. im Polnischen mit einer bestimmten Kategorie zum Ausdruck gebracht werden, wie zum Beispiel mit dem instrumentalen Kasus oder mit dem vollendeten bzw. unvollendeten Aspekt?

\section{WELCHE UNTERSCHIEDE MÜSSEN BEACHTET WERDEN?}

Die vorhandenen kontrastiven Grammatiken thematisieren den Vergleich von Sprachsystemen (Engel et al. 2000, deutsch-polnische bilaterale kontrastive Grammatik), ohne auf die Ursachen der Differenzen einzugehen. Der Unterschied liegt in der Bestimmung der Zielgruppe, bei Engel etwa - sowohl Deutsche als auch Polen mit Deutschkenntnissen. Die Autoren von anderen, nicht kontrastiv angelegten Grammatiklehrbüchern appellieren an die Ausländer im Allgemeinen (Helbig \& Buscha 1972), manchmal wird Englisch als Kontrastsprache herangezogen (Fandrich \& Thurmair 2018). Bis jetzt gibt es weder eine russisch-deutsche noch eine polnisch-deutsche kostrastive Grammatik. In der Grammatik der deutschen Sprache von Averina und Kostrova (2019) werden interkulturelle Unterschiede zwischen der deutschen und russischen Germanistik diskutiert, ohne dass die linguokulturelle Spezifik des Sprachgebrauchs des Russischen und des Deutschen systematisch berücksichtigt wird. In der geplanten Grammatik werden grammatische Kategorien der slawischen Sprachen aus der Perspektive der slawischen Muttersprachler systematisiert (vgl. Kostrova \& Prokop 2019). Wir berücksichtigen zwar zwei konkrete Sprachen: Russisch und Polnisch, doch viele Besonderheiten dieser Sprachen lassen sich auch auf andere slawische Sprachen übertragen. Diese Besonderheiten sind wie folgt:

In slawischen Sprachen gibt es bestimmte kategoriale Lakunen, die die Spezifik des deutschen Sprachbaus ausmachen und didaktisch am schwersten sind. Viele Schwierigkeiten bereiten russischen wie polnischen Studierenden solche Kategorien wie 
- der Artikelgebrauch: zahlreiche Fehler erscheinen dort, wo der Lernende zwischen dem bestimmten, dem unbestimmten und dem Nullartikel wählen muss, wozu er folgende Informationen braucht: Informationen über kontextuelle Beziehungen zwischen mehr oder weniger benachbarten Sätzen; Informationen über außersprachliche Situation; Informationen über die semantischen Verhältnisse im Satz sowie die Informationen über die grammatischen Verhältnisse im Satz (Grimm, 1986: 15). All diese Informationen werden in bisherigen Lehrwerken nicht präzise genug dargestellt, so dass die Determinatoren immer noch zahlreiche Fehler verursachen;

- schwache Deklination der Substantive: die Fehler resultieren wohl aus der Tatsache, dass die Flexion im Russischen und im Polnischen differenzierte Formen für einzelne Kasus aufweist, je nach Genus, Zahl und Auslaut des Substantivs, in der schwachen Deklination im Deutschen wird dieser Formenreichtum bis auf das Minimum reduziert;

- Deklination der Adjektive - in slawischen Sprachen entsprechen die Deklinationsendungen dem Genus, Kasus und der Zahl, im Deutschen ist die Präsenz des Artikels für die Wahl der Deklinationsendung maßgebend;

- der Elativ, insbesondere die Wahl zwischen dem bestimmten Artikel und dem Nullartikel;

- unpersönliche Sätze;

- Pronominaladverbien;

- analytische Vergangenheitsformen;

- Futur II;

- das System der Konjunktivformen, insbesondere der Konditionalis;

- manche Nebensätze.

Diese Themen bedürfen einer sorgfältigen didaktischen Bearbeitung. Einer ausführlichen Didaktisierung müssen auch Kategorien unterliegen, die in den slawischen Sprachen geringer funktional belastet sind: das Passiv (Unterscheidung zwischen dem Vorgangspassiv und dem Zustandspassiv), der Konjunktiv I, die Partikeln, die Komposita, Modalverben und Verben mit modaler Bedeutung. In diesem Fall müssen typische Kontexte gegeben werden, in welchen diese Kategorien im Gegenwartsdeutschen vorkommen.

Spezielle Übungen sollen den Unterschied im Gebrauch von allgemeinen (übergreifenden) Kategorien der slawischen Sprachen vs. differenzierte Kategorien des Deutschen trainieren. Das betrifft zum Beispiel das Possessivpronomen свой / swój oder manche Präpositionen $(n a, v, o)$.

Viele Schwierigkeiten bereiten den Lernern syntaktische Besonderheiten des Deutschen aus der Sicht des slawischen Sprachbaus: feste Wortstellung im Satz und in der Substantivgruppe mit erweiterten Attributen. Besonderer Beachtung 
verdienen auch semantische Unterschiede im Bereich der Konjunktionen (russ.: что, чтобы, когда, как, чем, poln. co, żeby, kiedy, jak, czym).

Der slawische Sprachbau lässt Übercharakterisierung zu, beispielsweise im Bereich der Polynegation und der Polyflexion. Die Mononegation des Deutschen muss didaktisch beachtet werden.

In slawischen Sprachen sind manche Kategorien funktional gewichtet vor dem Hintergrund des Deutschen, das sind beispielsweise reine Kasus, eingliedrige Sätze, Ellipsen in der gesprochenen Sprache. Die Lerner müssen entscheiden, wie diese Kategorien in der Zielsprache wiederzugeben sind. Die Grammatik sollte da verschiedene Ausdrucksmöglichkeiten zeigen, besonders, was gesprochene Sprache angeht.

\section{KOGNITIVE GRUNDLAGEN DER UNTERSCHIEDE}

Da die Zielgruppe einer erklärenden Grammatik Erwachsene sind, zum Teil pädagogisch orientiert, erscheint es zweckmäßig, bei der Beschreibung der grammatischen Kategorien von kognitiven Grundlagen auszugehen, die die Gestaltung von diesen Kategorien beeinflussen. Deshalb werden slawische und deutsche mentale Welten in ihren Grundzügen kontrastiv charakterisiert. Das Hauptgewicht liegt dabei auf der Beschreibung der kognitiven Wahrnehmung von Zeit und Raum, von Gegenstands- und Handlungswelten.

Die Zeit wird in slawischen Kulturen als Kreislauf wahrgenommen (Krasukhin 1997), in der deutschen Kultur aber als Linie (Schroll-Machl 2003: 87). Davon hängt der Unterschied im grammatischen Ausdruck dieser Kategorie ab: in slawischen Sprachen ist sie untrennbar mit der Kategorie des verbalen Aspekts verbunden. Dadurch werden Handlungen bzw. Ereignisse entweder in ihrer Totalität oder als dauernd dargestellt. Im ersten Fall kann man sie als einen geschlossenen Kreis vorstellen, im zweiten sind sie einem offenen Kreise ähnlich. Das hat weitgehende didaktische Folgen. In der Grammatik soll gezeigt werden, wie diese Zeitwahrnehmung auch die nominale Sphäre beeinflusst, und zwar den Artikelgebrauch. Andererseits muss man die Lerner auf die lineare Wahrnehmung der Zeit orientieren, die im deutschen Sprachbewusstsein vorhanden ist und die die Entwicklung der relativen Tempusformen herbeigerufen hat.

Die Wahrnehmung des Raums betrifft die Gegenstandswelt. Im deutschen Kulturstandard spielen die Direktheit und Pünktlichkeit eine wichtige Rolle (Schroll-Machl 2003: 83). Das bedingt eine genaue sprachliche Charakteristik der Gegenstände als definit bzw. indefinit. Der polnischen Kultur wird das Merkmal der unzulänglichen Kontrollierbarkeit zugeschrieben (Boski 2003: 126), in der russischen Kultur wird Vieles „in verschleierter Form [...] zum 
Ausdruck gebracht" (Schroll-Machl 2003: 109). Es ist wohl eines der Gründe, welcher die Charakteristik der Nomina in Bezug auf ihre Definitheit / Indefinitheit erübrigt. Der andere Grund hängt mit aspektuellen Eigenschaften der slawischen Verben zusammen. In slawischen Sprachen ist verbale Definitheit grammatikalisiert, deshalb bleibt die Kodierung nominaler Definitheit in diesen Sprachen unsichtbar (vgl. Leiss 2000: 268). Der perfektive Aspekt weist auf die Definitheit im verbalen Raum hin, die im Nomen nicht mehr kodiert wird. Die Indefinitheit lässt sich im Gegenteil mit dem imperfektiven Aspekt verbinden (Kashkin 2001).

Die Berücksichtigung dieser Tendenz hilft einige Fehler im Artikelgebrauch vermeiden. Es ist besonders wichtig, die genannten Tendenzen des Artikelgebrauchs den Lernern bereits in der Anfangsstufe bewusst zu machen. Eine Fehleranalyse der Aufsätze der russischen Studierenden aus dem ersten Studienjahr macht deutlich, dass sie den Artikelgebrauch mit dem verbalen Aspekt gar nicht assoziieren. So hat das Verb haben eine dauernde imperfektive Semantik, setzt folglich unbestimmte Objekte voraus. Die Studierende wählt aber im zweiten und dritten Satz den bestimmten Artikel, vgl.:

Ich möchte eine Dreizimmerwohnung haben, um dort mit meiner zukünftigen Familie zu wohnen. Wir hätten das Gästezimmer, das Schlafzimmer und das Kinderzimmer. Meine Wohnung muss auch das Telefon haben ${ }^{2}$.

Doch auch in der fortgeschrittenen Stufe entstehen bei Lernern oft Zweifel. Das belegen wir mit dem Beispiel einer polnischen Studentin aus dem Masterstudiengang, die in einem Satz beide Artikel gelassen hat, wo Nullartikel am Platze wäre:

Seit 5 Jahren habe ich ein das große Glück, mein Hobby zum Beruf machen zu dürfen.

Ein anderes Beispiel stammt auch aus dem Bewerbungsschreiben einer polnischen Studierenden. In dem folgenden Satz muss die Handlung eindeutig als abgeschlossen bewertet werden, was eine Ganzheit des direkten Objekts und somit seine Definitheit voraussetzt, die Studentin aber wählt den unbestimmten Artikel:

Während meines Studiums [...] habe ich ein Stipendium für einen Deutschkurs in Hamburg bekommen.

${ }^{2}$ Das Beispiel wurde den Aufsätzen entnommen, die mit den Studierenden der Universität Samara durchgeführt wurden. 
Das Beispiel gilt für die Lerner mit slawischer Muttersprache als Zweifelfall, weil diese immer die kommunikative Funktion des Objekts berücksichtigen und zwischen seiner Bestimmtheit als einer Ganzheit oder Unbestimmtheit als Hervorhebung wählen müssen. Eine spezielle Forschung zeigt (vgl. Kostrova 2019), dass sich deutsche Übersetzer aus dem Russischen circa in der Hälfte der Fälle für Bestimmtheit entscheiden, in der anderen Hälfte aber für Unbestimmtheit. Die Priorität der Unbestimmtheit muss aber begründet werden. Als gängige Begründungsursachen gelten mögliche Zählbarkeit des Objekts oder seine Einschätzung. Im angeführten Beispiel ist doch der bestimmte Artikel logischer, denn das direkte Objekt wird durch das nachgestellte Attribut identifiziert. Darüber hinaus betont der Gebrauch von Perfekt die Abgeschlossenheit der Situation und somit die Bestimmtheit sowohl des Zustandes als auch des Objekts, das dazu beigetragen hat.

\section{FAZIT}

Die Schwierigkeiten der erklärenden deutschen Grammatik für slawische Muttersprachler können durch folgende didaktische Ansätze aufgehoben bzw. reduziert werden:

1. Auswahl der zu beschreibenden grammatischen Phänomene, ausgehend von den abweichenden strukturell-semantischen und funktionalen Unterschieden im Polnischen und im Russischen vor dem Hintergrund des Deutschen.

2. Beschreibung der grammatischen Phänomene des Deutschen aus der Perspektive der slawischen Sprachen. Fokussierung auf die Themen, die besondere Schwierigkeiten im Spracherwerb bereiten, welche in den meisten Fällen auf der Fehleranalyse basiert.

3. Berücksichtigung des realen Gebrauchs in den Textsorten und mündlichen Kontexten, was einen Einblick in das Funktionieren der sprachlichen Einheiten auch für diejenigen Lerner verschafft, für welche reguläre Kontakte mit deutschen Muttersprachlern erschwert sind.

4. Fokussierung auf der linguokulturellen Spezifik der grammatischen Gestaltung von Textsorten und Gebrauchskontexten, die sich von den Textsorten und der linguokulturellen Spezifik der slawischen Kulturen unterscheiden. So plädieren C. Fandrich und M. Thurmair für eine textsortenbezogene Grammatikvermittlung. In einem ",didaktischen Fenster" führen sie Textsorten an, in denen das Passiv vorgezogen wird. Das sind nicht nur einfache Sachtexte, wo beispielsweise Arbeitsabläufe beschrieben werden, sondern auch wissenschaftliche Texte und Lexikonartikel, 
Leserbriefe und „Lebensbeschreibungen“, aber auch Instruktionen, Rezensionen und Gutachten (Fandrich \& Thurmair 2018: 62-64). Wenn die Lerner diese ganze Bandbreite sehen, muss ihnen die Bedeutung des Themas "Passiv" bewusst werden.

5. Angebot an Trainingsübungen, die berufliche Kompetenzen der Lerner entwickeln bzw. festigen helfen.

\section{LITERATURVERZEICHNIS}

Averina, A. V. / Kostrova, O. A. (2019). Grammatika nemeckogo jazyka. Moskau: JUrajt (na nemeckom jazyke).

Bimmel, P. / Rampillon, U. (2000). Lernerautonomie und Lernstrategien. München: Langenscheidt.

Boski, P. (2003). Polen. In: A. Thomas / S. Kammelhuber / S. Schroll-Machl (Hrsg.). Handbuch Interkulturelle Kommunikation und Kooperation. Bd. 2: Länder, Kulturen und interkulturelle Berufstätigkeit (S. 122-133). Göttingen: Vandenhoeck \& Ruprecht.

Duden (2007). Das große Fremdwörterbuch. Herkunft und Bedeutung der Fremdwörter. Stichwort: Lapsologie. 4. aktualisierte Auflage. München / Lepzig / Wien / Zürich: Dudenverlag.

Engel, U. et al. (2000). Deutsch-polnische kontrastive Grammatik. 2 Bände. Warszawa: Wydawnictwo Naukowe PWN.

Fandrich, C. / Thurmair, M. (2018). Grammatik im Fach Deutsch als Fremd- und Zweitsprache. Grundlagen und Vermittlung. Berlin: Erich Schmidt Verlag.

Grimm, H.-J. (1986). Untersuchungen zum Artikelgebrauch im Deutschen. Liepzig: VEB Verlag Enzyklopädie.

Grucza, F. (1993). Zagadnienia ontologii lingwistycznej: O językach ludzkich i ich (rzeczywistym) istnieniu. In: J. Bartmiński (Hrsg.). Opuscula Logopedica in Honorem Leonis Kaczmarek (S. 25-47). Lublin: UMCS.

Grucza, F. (1997). Języki ludzkie a wyrażenia językowe, wiedza a informacja, mózg a umysł ludzki. In: F. Grucza / A. Dakowska (Hrsg.). Podejście kognitywne w lingwistyce, translatoryce i glottodydaktyce (S. 7-21). Warszawa: WUW.

Helbig, G. / Buscha, J. (1972). Deutsche Grammatik: Ein Handbuch für den Ausländerunterricht. Leipzig: VEB Verlag Technik.

Kantorczyk, U. / Komorowska, E. / Lysakova, I. / Veselovskaja, T. (2008). Pragmatik von Aufforderungshandlungen im Deutschen, Polnischen und Russischen. Szczecin / Rostock: Print Group sp. z o.o.

Kashkin, V. B. (2001). Funkcionalnaja tipologija (Neopredelennyj artikl). Voronezh: VGTU.

Kieweg, W. (2007). Fehler erkennen - Fehler vermeiden. Der fremdsprachliche Unterricht Englisch 41 (88): Fehlerbewußtes Lernen, 2-11.

Kostrova, O. A. (2019). Strategii sozdaniya aspektualno-determinativnogo ravnovesiya v nemeckom i russkom yazykah. Voprosy kognitionoj lingvistiki, 3, 72-84.

Kostrova, O. A. / Prokop, I. (2019). Erklärende deutsche Grammatik für slawische Muttersprachler. GERMANISTIKA Nove et nova: Materialy Mieżdunarodnoj nauchno-prakticheskoj konferencji. 10-12 aprelja. $2019 \mathrm{~g}$. Moskva, 211-214. https:/ / linguanet.ru/upload/medialibrary/d27/d27afbe447937cb2202bd80bb82cfa5a.pdf [Zugriff am: 12.03.2020]. 
Krasukhin, K. G. (1997). Tri modeli indoevropejskogo vremeni na materiale leksiki i grammatiki. In: N.D. Arutyunova / T.E. Yanko (Hrsg.). Logicheskij analiz jazyka. Jazyk i vremja (S. 62-77). M.: „Indrik“: Rossijskaja Akademija Nauk.

Kroschewski, A. (2000). „False friends" und "true friends". Ein Beitrag zur Klassifizierung des Phänomens der intersprachlich-heterogenen Referenz und zu deren fremdsprachendidaktischen Implikationen. Frankfurt am Main: Peter Lang.

Leiss, E. (2000). Artikel und Aspekt. Die grammatischen Muster von Definitheit. Berlin et al.: De Gruyter. Lipczuk, R. / Mecner, P. / Westphal, W. (2000). Lexikon der modernen Linguistik. Ausgewählte Begriffe zur Kommunikation und Kognitionswissenschaft. 2.erweiterte Ausgabe. Szczecin: Wydawnictwo Promocyjne Albatros.

Schroll-Machl, S. (2003). Deutschland. In: A. Thomas / S. Kammelhuber / S. Schroll-Machl (Hrsg.). Handbuch Interkulturelle Kommunikation und Kooperation. Bd. 2: Länder, Kulturen und interkulturelle Berufstätigkeit (S. 72-89). Göttingen: Vandenhoeck \& Ruprecht.

Schroll-Machl, S. (2003). Russland. In: A. Thomas / S. Kammelhuber / S. Schroll-Machl (Hrsg.). Handbuch Interkulturelle Kommunikation und Kooperation. Bd. 2: Länder, Kulturen und interkulturelle Berufstätigkeit (S. 90-112). Göttingen: Vandenhoeck \& Ruprecht.

Wolff, D. (2003). Lernerautonomie und selbstgesteuertes fremdsprachliches Lernen. Überblick. In: K. Bausch / K.-R. Christ / H.-J. Krumm (Hrsg.). Handbuch Fremdsprachenunterricht (S. 321-326). Tübingen / Basel: Francke.

Zabrocki, L. (1980). Grundfragen der konfrontativen Grammatik. In: L. Zabrocki (Hrsg.). U podstaw struktury i rozwoju jezyka. At the foundation of language structure and development (S. 254-270). Warszawa / Poznań: Państwowe Wydawnictwo Naukowe.

Received: 6.22.2019; revised: 25.02 .2020

OLGA A. KOSTROVA

Samarskij gosudarstvennyj socialno-pedagogicheskij universitet

olga_kostrova@pgsga.ru

ORCID: 0000-0002-2044-6048

IZABELA PROKOP

Uniwersytet im. Adama Mickiewicza w Poznaniu

iprok@amu.edu.pl

ORCID: 0000-0002-4933-1789 
